\title{
DESENVOLVIMENTO DE UM PROGRAMA DE PROMOÇÃO DA SAÚDE PARA TRABALHADORES ADMINISTRATIVOS
}

\section{José Adolfo Menezes Garcia Silva}

Mestre em Desenvolvimento Humano e Tecnologias, pela Universidade Estadual Paulista, Brasil.

\section{Tássia Tamy Hirono Hotta}

Graduada em Fisioterapia, pela Universidade Federal do Triângulo Mineiro (UFTM), Brasil.

\section{Thiago Henrique da Silva}

Fisioterapeuta. Programa de Pós-graduação em Ciências da Reabilitação da Faculdade de Medicina da Universidade de São Paulo (FMUSP), Brasil.

\section{Maria Helena Morgani de Almeida}

Doutora, Docente Doutora do Curso de Terapia Ocupacional da Faculdade de Medicina da Universidade de São Paulo (FMUSP) e Docente do Programa de Pós-graduação em Ciências da Reabilitação da FMUSP, Brasil.

\section{Fátima Aparecida Caromano}

Doutora, Docente Doutora do Curso de Fisioterapia da Faculdade de Medicina da Universidade de São Paulo (FMUSP) e Docente do Programa de Pós-graduação em Ciências da Reabilitação da FMUSP, Brasil.

E-mail: fcaromano@uol.com.br
RESUMO: Este estudo objetivou aplicar um programa educacional e intervencional de fisioterapia visando melhorias na qualidade de vida de funcionários administrativos. Participou do estudo um grupo de 13 funcionários, avaliados pelo questionário SF-36, pelo o índice de incapacidade de Oswestry e pelo acompanhamento do desempenho durante as atividades físicas. O protocolo de intervenção foi composto por atividades físicas, aulas ministradas por profissionais da saúde e atividades sociais-recreacionais, que ocorreram ao longo de dois meses. Também foram realizadas orientações ergonômicas de posto de trabalho, de forma individualizada. A análise estatística foi realizada por meio do programa GraphPadPrism ${ }^{\circledR}$. Foi encontrada diferença significativa $(p=0,03)$ entre a pontuação inicial média obtida no SF-36, com melhorias pronunciadas nos domínios "Vitalidade" $(p<0,01)$ e "Aspectos sociais" $(p=0,05)$. O programa de promoção de saúde incrementou a qualidade de vida dos trabalhadores, com desenvolvimento de qualidades físicas e aspectos sociais. A orientação profissional foi fundamental neste processo. O programa possibilitou maior conhecimento sobre saúde e incentivo à prática de atividades físicas.

PALAVRAS-CHAVE: Trabalho; Qualidade de vida; Saúde do trabalhador; Modalidades de fisioterapia.

\section{DEVELOPMENT OF A HEALTH-ENHANCEMENT PROGRAM FOR MANAGERS}

\begin{abstract}
An educational and interventional program in physiotherapy was applied to thirteen managers to improve their life quality. They were evaluated by Questionnaire SF-36, Oswestry Disability Index and by follow-up of performance during physical activities. Intervention protocol comprised physical activities, lectures by health professionals and social-recreational activities, throughout two months. Individual ergonomic guidelines on work were also undertaken. Statistical analysis was done with GraphPadPrism ${ }^{\circledR}$. There was significant difference $(p=0.03)$ between initial mean scores by SF-36, with great improvements in domains "Vitality" $(p<0.01)$ and "Social Aspects" $(p=0.05)$. Health enhancement program increased life quality of the workers, with the development of physical qualities and social aspects. Professional orientation was basic in the process. The program provided information on health and incentives for physical activities.
\end{abstract}

KEY WORDS: Labor; Life quality; Worker's health; Physiotherapy modalities. 


\section{INTRODUÇÃO}

A relação entre trabalhador e trabalho tem influência na saúde e na satisfação de forma geral, podendo ter impacto relevante na sua vida (D'ANGELO et al., 2016). Os fatores que reduzem a qualidade de vida (QV) estão relacionados com suas necessidades de saúde percebidas, sendo assim, quando identificadas situações adversas, devem ser tomadas medidas para melhoria das condições laborais (JUNG et al., 2017). O exercício físico pode aumentar os recursos mentais e diminuir a tensão física diária em ambiente de trabalho (RUTANEN et al., 2014). Minimizar a exposição ocupacional ao estresse físico e psicológico exige uma avaliação eficaz e perspectiva de ações por meio de programas de gerenciamento de grupo e pessoal (D'ETTORRE; GRECO, 2016).

A piora da qualidade de vida e o adoecimento podem acometer os trabalhadores sem que estes tenham ciência da real deterioração da própria saúde (PEREIRA, 2017). Desenvolver ações para orientação e promoção de saúde e QV para funcionários tem o intuito de garantir o aumento da produtividade sem sobrecarregar o funcionário. De forma geral, trata-se de uma estratégia que possibilita visualizar quais adequações são necessárias para a promoção do bem-estar do funcionário e da empresa (SEIDL; ZANNON, 2004).

Parte dos funcionários, geralmente, atua em áreas que impõem o uso prolongado da postura sentada em trabalhos utilizando computadores. Sabese que esta atividade, em médio e longo prazo, afeta o sistema musculoesquelético, cardiovascular e pulmonar (WAONGENNGARM; RAJARATNAM; JANWANTANAKUL, 2015). As queixas mais frequentes são de dores lombares, cervicais e nas pernas (AKKARAKITTICHOKE; JANWANTANAKUL, 2017). Quando o sistema cadeiramesa-funcionário não está ergonomicamente adequado ocorre o uso de posturas inadequadas com sobrecarga de peso assimétricas e maior ativação muscular para estabilização corporal, resultando em pressão significantemente maior na coluna (ZENK et al., 2012). A correção ergonômica é essencial e a prática de exercícios físicos com finalidade de fortalecer a musculatura pode minimizar as dores geradas pela atividade (JUNIO, 2015)

Com base nos fatos apresentados, o presente estudo buscou elaborar, aplicar e avaliar as respostas a um programa educacional intervencional fisioterapêutico visando a melhoria da qualidade de vida de funcionários administrativos.

\section{MÉTODO}

Estudo quase-experimental com delineamento de pré e pós-teste, composto de amostragem consecutiva. Foram incluídos no estudo funcionários administrativos de um departamento específico de uma universidade pública do Estado de São Paulo. Estes participantes poderiam ser de ambos os sexos, sem restrições quanto à idade. Foram excluídos da amostra indivíduos com distúrbios cardiovasculares, ortopédicos, neurológicos ou de qualquer outra natureza que inviabilizassem a prática das atividades oferecidas. Os participantes do estudo assinaram o Termo de Consentimento Livre Esclarecido. O estudo foi aprovado pelo Comitê de Ética em Pesquisa da Faculdade de Medicina da Universidade de São Paulo (processo CAAE: 22106113.9.000.0065).

Todos os participantes responderam um questionário para gerar o perfil sociocultural básico, constando de dados pessoais, como número de telefone de contato, sexo, idade, data de nascimento, queixas de saúde e a função laboral exercida no setor administrativo, sendo de conhecimento prévio o fato que estas funções impõem que o trabalho seja realizado na postura sentada por períodos de 8 horas por dia, com pequenos intervalos para café e deslocamento até banheiro ou outros postos de trabalho. Esta característica é um dos motivos para que se estimulem programas que incentivem mudanças de hábitos e promovam melhoras na saúde e na qualidade de vida. Os funcionários que participaram do estudo exerciam a mesma atividade há, pelo menos, oito anos.

Os participantes foram avaliados quanto sua qualidade de vida autopercebida, por meio do questionário Medical Outcomes Study 36 - Item Short Form Health Survey (SF-36). Este questionário foi criado por Ware e Sherbourne (1992), traduzido e validado para o português por Ciconelli et al. (1999), e é um instrumento de fácil aplicação e compreensão. O SF-36 é um questionário genérico que avalia a $\mathrm{QV}$ de forma multidimensional, e é composto por 36 itens abrangendo 
as áreas de capacidade funcional (CF), aspectos físicos (AF), dor, estado geral de saúde (EGS), vitalidade (Vit), aspectos sociais (AS), aspectos emocionais (AE) e saúde mental (SM). O escore total para cada domínio varia de zero a 100 , sendo que, quanto mais próximo de zero, pior condição de QV e, quanto mais próximo de 100, melhor condição de QV.

Especificamente para os funcionários que relataram lombalgia, foi aplicado o questionário de índice de incapacidade de Oswestry (IIO), desenvolvido por Fairbank et al. (1980), traduzido e validado para a língua portuguesa do Brasil por Vigatto et al. (2007). Este questionário é constituído por dez sessões que se referem às atividades diárias que podem ser interrompidas ou prejudicadas pela lombalgia. Cada uma das sessões contém seis afirmações, as quais, progressivamente, descrevem maior grau de dificuldade na atividade que a afirmação precedente. A classificação final do IIO é dada referente à porcentagem obtida respectivamente: nenhuma disfunção (0\%), disfunção mínima (1 a 20\%), disfunção moderada (21 a 40\%), disfunção severa (41 a $60 \%$ ), incapacidade severa (60 a $80 \%$ ) e participante acamado ou com hipersomatização dos sintomas (acima de 81\%) (MASSELLI et al., 2007).

O desempenho durante as atividades físicas foi realizado em três momentos: ao iniciar as atividades, durante a quarta sessão e na última sessão. Precedendo e após o término de cada sessão de atividade física os participantes passaram por medição da pressão arterial, procedimento que informa sobre o estado cardiovascular do paciente, a resposta à atividade física e direciona a aplicação da sessão de exercícios, coletada segundo normas específicas para fisioterapeutas (FRESE; FICK; SADOWSKY, 2011). Ao término do programa foi recolhido um questionário destinado a avaliar as atividades desenvolvidas pelo programa com espaço para sugestões, críticas e elogios.

O programa foi oferecido duas vezes por semana, em sessões de 30 minutos, ao longo de dois meses, totalizando 18 sessões, todas realizadas durante o horário de trabalho. Foram formados grupos de até seis pessoas. Os funcionários puderam escolher o horário em que as atividades seriam realizadas, conforme necessidades pessoais e critérios de trabalho. Foi dada a orientação para que os participantes escolhessem o horário de menos sobrecarga laboral para que o setor continuasse em funcionamento e utilizassem revezamento na saída dos funcionários do posto de trabalho, para não esvaziar setores.

O programa foi organizado em três tipos de sessões.

Um tipo foi elaborado visando atividades físicas divididas de alongamento, força e treino aeróbico, por oito sessões, sendo uma por semana. Nestas, os participantes eram instruídos sobre a forma correta de realizarem os exercícios físicos simples e incentivados a dar prosseguimento nas atividades em suas casas. Para garantir a recordação das informações fornecidas e treinadas, foi elaborado um manual contendo fotos e informações sobre os exercícios que poderiam ser feitos em casa, em número pequeno visando adesão.

Para a realização do exercício de alongamento foi preconizado que o participante permanecesse na posição sentada no chão com as pernas unidas e estendidas, realizasse uma inspiração máxima seguida de expiração em frenolabial associado de flexão anterior de tronco, levando as mãos em sentido aos pés e permanecesse por pelo menos 30 segundos em posição de alongamento, sustentar o peso do corpo pelos apoios de antebraços e pontas dos pés, realizando contração do músculo transverso do abdome, multifidos e assoalho pélvico, durante o tempo de cadeias posteriores de membros inferiores. Os exercícios de força utilizaram o corpo como em exercícios como sustentar a perna em flexão durante decúbito dorsal. Para executar o exercício aeróbico, os participantes deveriam ficar em decúbito dorsal com antebraços apoiados no chão e elevação de pernas a aproximadamente $30^{\circ}$ simulando o pedalar de uma bicicleta com movimentos circulares amplos e flexão dorsal e plantar dos pés, progredindo de acordo com o número de repetições suportados. Estes exercícios são simples e utilizados em programas clássicos, na Yoga e até no método Pilates.

O segundo tipo de encontro consistiu na exposição de quatro aulas didáticas ministradas por profissionais da área da saúde sobre os seguintes temas: "Descarte de medicamentos", "Prevenção de LER/DORT por meio das técnicas de conservação de energia", "A 
importância de se hidratar" e "Controle de emoções", com periodicidade quinzenal e com duração de 45 minutos. Foram utilizados slides em Power Point para suporte das atividades

A terceira intervenção foi a realização de atividades sociais e recreativas em quatro encontros. Ocorreram três sessões de cinema, exibindo curtas metragens de comédias com enredos baseados em aspectos morais, apresentados em televisão de tela grande e uma dinâmica de grupo com o objetivo de conscientizar o grupo sobre as dificuldades enfrentadas em cada posto de serviço, com periodicidade quinzenal, com duração de 45 minutos.

O primeiro encontro foi utilizado para explicar a proposta do estudo aos funcionários e realizar o procedimento de avaliação inicial completo. No quarto encontro, destinado à atividade física, foi realizada uma avaliação do desempenho obtido nos exercícios físicos, focando no número de repetições em tempo préestabelecido. Então, no último encontro foi realizada a reavaliação completa dos participantes.

Conjuntamente com estas atividades todos os postos de serviço foram visitados e quando necessário, foram realizados ajustes ergonômicos, tais como adequações na distribuição dos objetos sobre a mesa, regulagem da altura de monitores, cadeiras e apoio de braço e oferecido apoios de pés para os participantes que necessitavam desta ação.

A análise estatística do número de exercícios por período de tempo fixo e os escores nos questionários foi realizada por meio do programa GraphPadPrism ${ }^{\circledR}$ versão 5. Todas as variáveis foram avaliadas quanto à normalidade de sua distribuição por meio do teste Shapiro-Wilk. Para a análise do índice de correlação interna foi utilizado o teste de Sperman, as comparações entre os escores iniciais e finais do questionário SF-36 foram realizados por meio do teste de Wilcoxon. As comparações entre as três avaliações referentes ao desempenho no programa de atividade física foram realizadas por meio do teste de Fredman e, posteriormente, avaliado pelo teste Post hoc de Dunns. A comparação entre o momento inicial e final do IIO foi realizada por meio do teste T de Student para amostras pareadas. Foi considerado como satisfatório um índice de significância com magnitude de $5 \%(p \leq 0,05)$. Para a verificação de possíveis correlações entre as variáveis, foi utilizada, como referencial, a classificação de Munro (2005), em que: valor de $r$ baixo, de 0,26 a 0,49 ; moderado, de 0,50 a 0,69 ; alto, de 0,70 a 0,89 ; e muito alto, de 0,90 a 1,00 .

\section{RESULTADOS}

No departamento avaliado há 24 funcionários administrativos, destes 16 funcionários se inscreveram para participar do programa $(66,7 \%)$, sendo que finalizaram o programa 13 funcionários (81,3\%). O grupo final era composto de cinco homens com idade média de

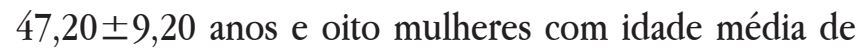
$36 \pm 8,5$ anos a média de idade geral foi de 40,30 $\pm 10,5$ anos. A frequência dos participantes durante as atividades físicas foi em média de $65,8 \pm 26,6 \%$, nas atividades sociais foi de $69,2 \pm 23,6 \%$.

O escore total médio obtido no questionário SF-36, pelos participantes na avaliação inicial, foi de $55,98 \pm 18,75$ pontos. Na Tabela 1 é mostrado o Índice de Correlação Interno Inicial do questionário SF-36, assim como a média e o desvio-padrão obtidos em cada um dos domínios. Na avaliação final o escore foi de $69,5 \pm 10,5$ pontos.

Por meio da comparação entre os escores inicial e final obtidos no questionário SF-36, encontramos diferença estatisticamente significante para a pontuação total $(p=0,03)$ e para os domínios "Vitalidade" $(p<0,01) \mathrm{e}$ "Aspectos Sociais" $(p=0,05)$. A Figura 1 mostra os gráficos em bloxplots dos escores iniciais e finais da análise dos dados oriundos do questionário SF-36 representando cada domínio no início e ao final (pontuação total). 
Tabela 1. Índice de Correlação Interna Inicial e Final do questionário SF-36

\begin{tabular}{lcccccc}
\hline & pi & ri & Média/Desvpadi & pf & Rf & Média/Desvpadf \\
\hline Capacidade funcional & $p<0.01$ & 0,84 & $68,75 \pm 25,39$ & $p<0,01$ & 0,92 & $77,69 \pm 19,85$ \\
Limitação por aspectos físicos & $p<0.01$ & 0,74 & $73,43 \pm 33,50$ & 0,21 & 0,37 & $82,69 \pm 27,73$ \\
Dor & $p<0.01$ & 0,84 & $49,68 \pm 19,84$ & $p<0,01$ & 0,77 & $57,07 \pm 15,06$ \\
Estado geral de saúde & 0.01 & 0,61 & $54,75 \pm 18,40$ & $p<0,01$ & 0,36 & $54,69 \pm 13,78$ \\
Vitalidade & $p<0.01$ & 0,78 & $32,81 \pm 19,40$ & $p<0,01$ & 0,83 & $61,53 \pm 15,86$ \\
Aspectos sociais & $p<0.01$ & 0,72 & $64,06 \pm 25,36$ & 0,09 & 0,47 & $79,80 \pm 17,33$ \\
Limitação por aspectos & $p<0.01$ & 0,69 & $54,16 \pm 40,13$ & 0,34 & 0,28 & $76,92 \pm 31,57$ \\
emocionais & $p<0.01$ & 0,75 & $50,25 \pm 19,37$ & 0,07 & 0,5 & $65,53 \pm 20,16$ \\
Sáude mental & & &
\end{tabular}

pi: Valor inicial de $p$; ri: Valor inicial de r; Média/Desvpadi: Média e Desvio-Padrão inicial; pf: Valor final de $p$; rf: Valor final de r; Média/Desvpadf: Média e Desvio-padrão final.

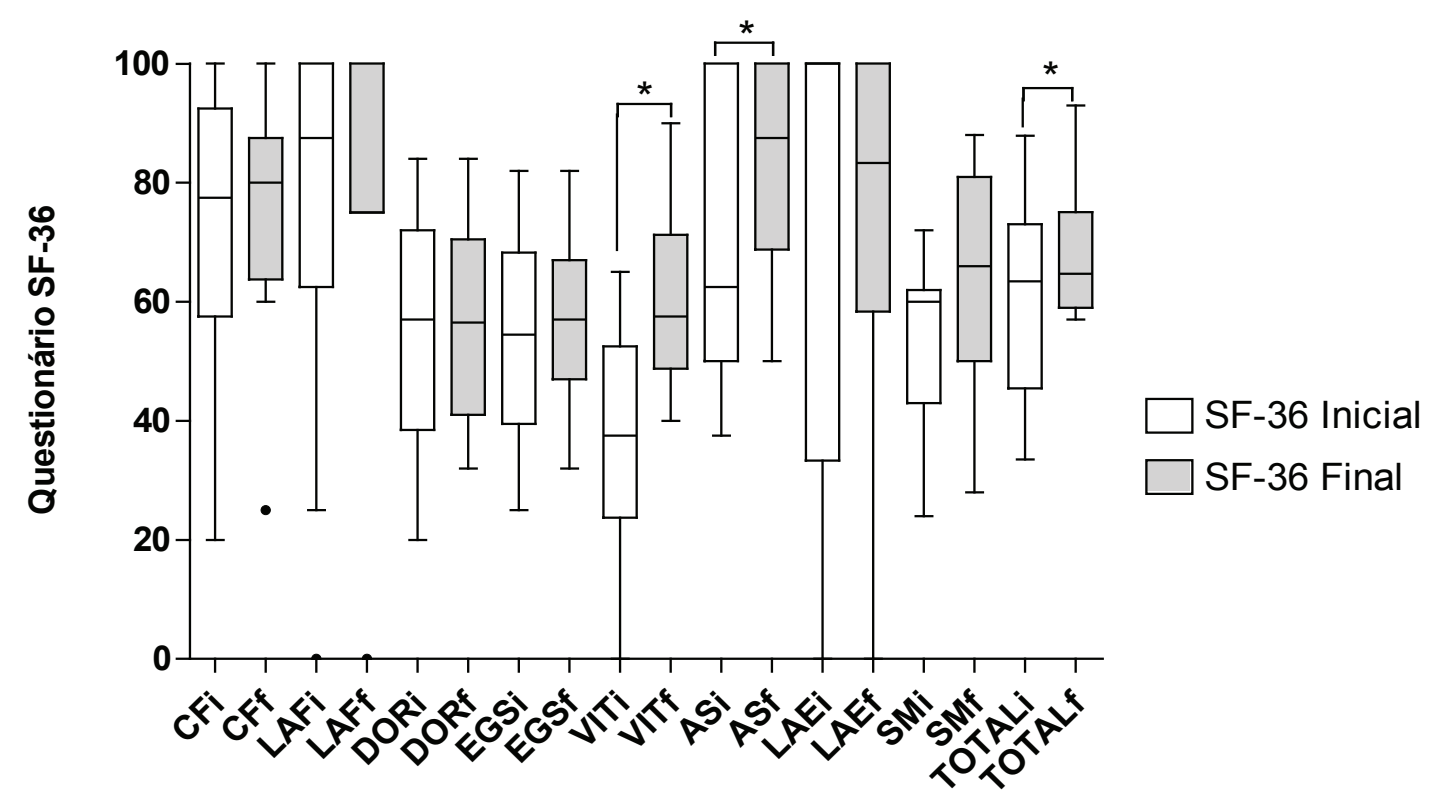

Figura 1. Bloxplots dos escores iniciais e finais do QdV SF-36.

*: Valor estatisticamente significante; CFi: Capacidade funcional inicial; CFf: Capacidade funcional final; LAFi: Limitação por aspectos físicos inicial; LAFf: Limitação por aspectos físicos final; DORi: Dor inicial; DORf: Dor fianl; EGSi: Estado geral de saúde inicial; EGSf: Estado geral de saúde final; VITi: Vitalidade inicial; VITf: Vitalidade final; ASi: Aspectos Sociais inicial; ASf: Aspectos Sociais final; LAEi: Limitação por aspectos emocionais inicial; LAEf: Limitação por aspectos emocionais fianl; SMi: Sáude mental inicial; SMf: Sáude mental fianl; TOTALi:

Total inicial; TOTALf: Total final. 
Os resultados apresentam maior similaridade na distribuição dos dados com consequente diminuição da amplitude entre os valores mínimos e máximos e aumento dos valores mínimos para todos os domínios exceto o domínio "Limitação por Aspectos Emocionais".

Os valores de mediana finais apresentaram variação com tendência ao acréscimo dos valores explicitados principalmente nos domínios "Vitalidade", "Aspectos Sociais" e discretamente para os domínios "Capacidade funcional", "Estado geral de saúde" e "Saúde Mental".

$\mathrm{Na}$ Figura 2 é mostrado, em boxsplots, o comportamento das avaliações de desempenho nas atividades físicas. Foi possível identificar diferenças estatisticamente significantes entre a primeira e a terceira avaliação para os exercícios de alongamento $(p<0,01)$ e aeróbico $(p<0,01)$. Não foram observadas diferenças estatisticamente significantes para o desempenho no exercício de força muscular $(p=0,06)$.

$\mathrm{Na}$ Figura 2 é apresentada a tendência ao crescimento no desempenho para os três grupos de exercícios analisados. O comportamento destas variáveis mostra aumento nos valores das medianas, assim como diminuição na amplitude entre as linhas de contenção inferior e superior, indicando menores discrepâncias entre o desempenho dos participantes.
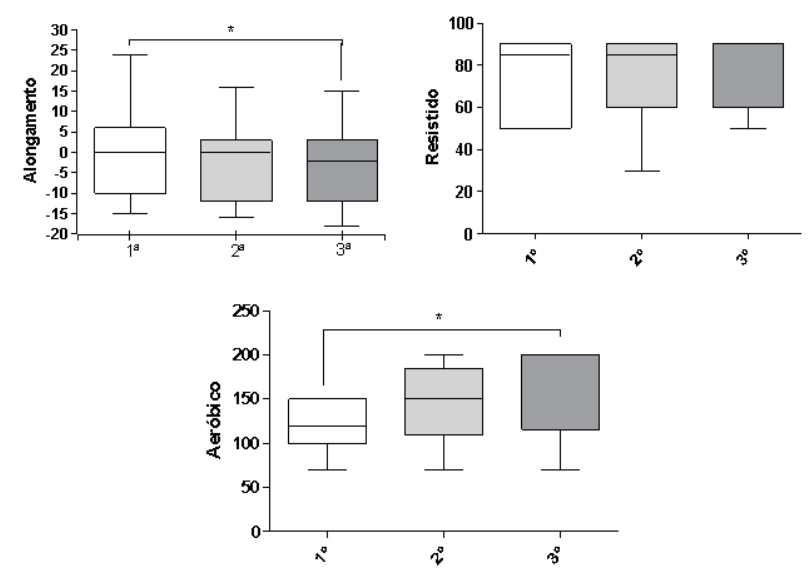

Figura 2. Bloxplots referentes ao desempenho no programa de atividade física

*: Diferença estatisticamente significante; $1^{\mathrm{a}}$ : Primeira avaliação; $2^{\mathrm{a}}$ Segunda avaliação; $3^{a}$ : Terceira avaliação

Dos 13 funcionários participantes do estudo, sete deles $(53,84 \%)$ apresentavam queixas de dores lombares não específicas. Durante a avaliação inicial, a pontuação média adquirida no IIO foi de $16,0 \pm 15,8 \%$ de comprometimento e após o protocolo de intervenção a pontuação média foi de $13,6 \pm 10,8 \%$ de comprometimento. Mesmo não ficando estabelecidas as diferenças estatisticamente significantes $(p=0,48)$, a observação da Figura 3 evidencia tendência de redução da amplitude dos valores das linhas de contenção inferior e superior comparando-se a avaliação inicial com a final, assim como a redução do quartil superior final indicando queda no nível de comprometimento máximo, e quartil inferior final tendo origem no nível 0 , indicando que algum dos participantes deixou de sentir dores lombares após o protocolo de intervenções.

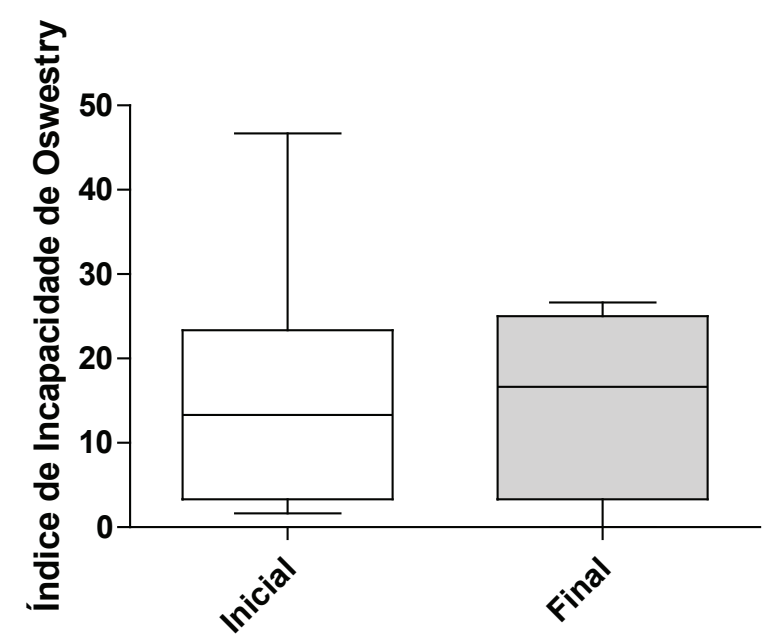

Figura 3. Bloxplots referentes às avaliações do índice de incapacidade de Oswestry

\section{DISCUSSÃO}

Os esforços de promoção da saúde no ambiente de trabalho buscam motivar os trabalhadores, reduzir o estresse laboral, aumentar a satisfação no trabalho e acarretam melhoria da qualidade de vida (TUNG et al., 2017). Com o objetivo de adaptar este contexto ao cenário da instituição estudada, este estudo elaborou um modelo de programa de promoção de saúde que poderá ser aplicado para diferentes grupos de funcionários, especialmente os que utilizam a postura sentada por períodos de tempo prolongados.

Medidas simples como ações educacionais de saúde com aconselhamento e autogestão demonstram 
impacto positivo na saúde e redução do estresse de trabalhadores. (ELLIOT; SHELLEY, 2005). No entanto, é fundamental que as empresas conduzam o gerenciamento de seus funcionários fundamentado em melhoria de seus hábitos de vida, com planejamento, ambientes saudáveis e envolvimento de pessoas, superando os obstáculos organizacionais (KIM; PARK; PARK, 2016).

Os projetos que propõem ganho de $\mathrm{QV}$ dentro do ambiente de trabalho devem estar baseados em ações, de preferência, multiprofissionais focadas em garantir o máximo desempenho do trabalhador, assegurando a manutenção de uma condição de saúde adequada (FISKER et al., 2013). Ao introduzir ações baseadas em diferentes campos do saber por meio do programa aqui proposto, foi observada melhora estatisticamente significante na QV dos participantes $(p=0,03)$.

As iniciativas de programas de intervenção no ambiente de trabalho demonstram efetivas para o ganho de QV de trabalhadores, potencializam o ganho de produtividade associado à prevenção do aparecimento de doenças e previnem a cronificação dos distúrbios presentes (DEL POZO-CRUZ et al., 2012). Durante o desenvolvimento deste estudo foi possível diagnosticar dois funcionários com níveis de pressão arterial elevados, que não sabiam desta alteração, e foram orientados e acompanhados na procura de assistência médica especializada.

Ao analisar o índice de correlação interno do questionário SF-36 foi encontrado que tanto no início quanto ao final do estudo, o domínio "Capacidade Funcional" apareceu como um dos domínios mais relacionados ao quadro geral de $\mathrm{QV}$ (respectivamente $\mathrm{r}=0,84$ e $\mathrm{r}=0,92)$. Houve troca de posição do domínio "Dor", que aparecia como segundo mais relevante inicialmente $(\mathrm{r}=0,84)$, pelo domínio "Vitalidade" $(\mathrm{r}=0,83)$. Assim, os resultados do presente estudo demonstram que esta troca representa uma alteração nos fatores que determinavam a $\mathrm{QV}$ nos momentos inicial e final do programa, indicando que o domínio "Dor" passou a ser menos impactante.

Foram evidenciados aumentos significativos nos domínios "Vitalidade" $(p<0,01)$ e "Aspectos Sociais" $(p=0,05)$. O domínio "Vitalidade" destina-se a representar o nível de esgotamento físico dos participantes e, o domínio "Aspectos Sociais", mede a interação com familiares, amigos ou qualquer outro grupo social ao qual faça parte. Os resultados do presente estudo sugerem que estes benefícios podem estar relacionados à participação no programa, em seu conjunto de atividades físicas, educativas e recreacionais, mesmo que breves e simples.

Outro aspecto a ser considerado nos nossos resultados é a diminuição dos quadros de dor lombar, que se associam diretamente com a produtividade e a $\mathrm{QV}$, além do fato que sua identificação em estágios iniciais pode impedir sua progressão (GANESAN et al., 2017).

Entre os 13 funcionários participantes sete apresentavam queixas de dores lombares. A dor lombar é altamente prevalente, sendo um obstáculo considerável para os pacientes, suas famílias e a sociedade (HENSCHKE et al., 2017). Em geral, a lombalgia impõe um ônus econômico significativo aos governos com custos elevados no sistema de saúde, dias de trabalho perdidos, redução da produtividade e aumento da incapacidade (GOUVEIA et al., 2016). Estima-se que até $85 \%$ da população em geral apresentará dor lombar em algum momento da vida e mais de $10 \%$ sofrerão de dor crônica duradoura (HENSCHKE; KAMPER; MAHER, 2015). Desenvolver episódios de lombalgia pode ter relação direta com a execução de atividades laborais na postura sentada, quando mantida por longos períodos de tempo, associado à não prática de atividades físicas (BARROS; ANGELO; UCHOA, 2011).

Em média, até 95,2\% dos funcionários que desenvolvem atividades laborais na postura sentada relatam desconfortos na região dorsal da coluna, com prevalência em trabalhadores acima dos 40 anos, em indivíduos sedentários e nos que trabalham há mais tempo na mesma instituição (FREITAS et al., 2011).

Exercícios físicos terapêuticos, atividades educacionais e sociais-recreativas foram combinados num único programa de promoção de saúde no presente estudo, de forma minimalista, simples e sem nenhuma forma de ônus para a instituição. Os benefícios advindos desta ação, focando no servidor como uma pessoa com diferentes necessidades, são suficientes para justificar esta forma de programa, como já defendido por outros estudos similares, mas com populações diferentes 
(RUTANEN et al., 2014; JUNIO, 2015; TUNG et al., 2017). Diferentes tipos de exercícios podem beneficiar os trabalhadores de formas distintas e as organizações devem incentivar seus funcionários a acessar programas regulares de exercícios (BRETLAND; THORSTEINSSON, 2015). Intervenções no ambiente de trabalho devem ocorrer com o devido preparo, mediante agendamento e planejamento, além da consideração das possibilidades e necessidades físicas do grupo. Se esta ação for combinada com a educação e estímulos em temas complementares, a exemplo de nutrição e aderência à prática de exercícios, certamente os resultados a serem obtidos serão mais relevantes (LUCKHAUPT et al., 2014).

Programas realizados em grupos tendem a apresentar melhores resultados nos aspectos sociais, como encontrado no presente estudo, e esta forma de organização de intervenção, ou seja, em grupo, é tida como a mais adequada para este fim (BURGARD; LIN, 2013). A participação em sessões de atividade física em grupo também demonstra redução no estresse percebido e melhora na QV física, mental e emocional, quando comparada com a prática de exercício regular individual (YORKS; FROTHINGHAM; SCHUENKE, 2017).

É fundamental, ao se pensar em promoção de saúde de trabalhadores, lembrar que o emprego está ligado à saúde de maneira positiva, como fonte de renda ou de outros benefícios materiais, além de ser fonte de integração social e reconhecimento (BURGARD; LIN, 2013).

Ao término do programa todos os 13 participantes avaliaram como positiva a interação entre os funcionários de diferentes setores e a prática de atividades físicas. As sessões de filmes breves com pipoca foram consideradas muito alegres e positivas. Os pontos negativos, enumerados por 12 dos 13 funcionários, foram vinculados à dificuldade de conciliar a demanda de trabalho com as pausas necessárias para a participação no programa. Questionados sobre sugestões para ampliação do programa, os funcionários elegeram atividades como caminhadas ao ar livre, sugerida por cinco participantes e atendimentos com massagem, sugerido por nove funcionários, além de orientação contínua de serviço de nutrição, sugerido por seis funcionárias, e cabe uma nota - todas com peso classificado como obesidade.
Este estudo corrobora plenamente com a afirmação de que intervenções educacionais multiprofissionais e o treinamento de postura ergonômica são ações eficazes para reduzir a dor lombar causada por atividades laborais (SHOJAEI et al., 2017).

A amostra deste estudo é pequena, tornando-se um fator limitante de pesquisa, mas ao mesmo tempo, permite mostrar que o trabalho em grupos pequenos pode ser mais enfático e pessoal, além de permitir maior troca entre os participantes. A avaliação de uma única categoria profissional evidencia informações mais precisas e direcionamentos mais específicos, podendo gerar indicadores sobre a intervenção a serem propostas por gestores. Para estudos futuros, sugerimos a expansão deste programa a diferentes grupos profissionais e a análise de variáveis não abordadas na presente pesquisa, como tempo de permanência na postura sentada durante o trabalho, evolução da composição corporal e a interação social entre membros do grupo. Estes estudos devem, prioritariamente, estar inseridos em políticas públicas nacionais de promoção de saúde de trabalhadores.

Considerando a Política Nacional de Promoção da Saúde (PNPS), o presente estudo insere-se em eixos prioritários como formação e educação, além de práticas corporais e atividade física (BRASIL, 2014). Este formato de programa evidencia uma tendência que pode gerar avanços expressivos na redução de fatores de risco para mortalidade da população mundial, relacionados à inatividade física (MALTA et al., 2014). Desta maneira, tornam-se fundamentais estudos que demonstrem a promoção da saúde e prevenção de doenças baseados na PNPS, contribuindo de forma efetiva para a melhoria das condições de saúde da população brasileira e propagando maior entendimento sobre este tema (ALVES; DOMINGUEZ UGÁ; PORTELA, 2016).

\section{CONCLUSÃO}

A adesão a um programa de promoção de saúde com diferentes atividades incrementou positivamente a qualidade de vida dos trabalhadores administrativos participantes, com ganho pronunciado evidenciado pelos domínios "Vitalidade" e "Aspectos Sociais" do SF- 
36 e contribuiu no desenvolvimento de qualidades físicas como o alongamento e a capacidade aeróbica, tendo o fisioterapeuta como mediador da qualidade de vida no ambiente de trabalho. Portanto, o programa pesquisado mostrou potencial para possibilitar o desenvolvimento do conhecimento sobre saúde e incentivo à prática de atividades físicas em trabalhadores administrativos.

\section{REFERÊNCIAS}

AKKARAKITTICHOKE，N.; JANWANTANAKUL，P. Seat Pressure Distribution Characteristics During 1 Hour Sitting in Office Workers With and Without Chronic Low Back Pain. Safety and health at work, 2017.

ALVES, D. C.; DOMINGUEZ UGÁ, M. A.; PORTELA, M. C. Promoção da saúde, prevenção de doenças e utilização de serviços: avaliação das ações de uma operadora de plano de saúde brasileira. Cadernos Saúde Coletiva, v. 24, n. 2, 2016.

BARROS, S. S.; ANGELO, R. C. O.; UCHOA, E. P. B. L. Lombalgia ocupacional e a postura sentada. Rev. dor, São Paulo, v. 12, n. 3, p. 226-230, Sept. 2011.

BRASIL. Política Nacional de Promoção da Saúde. Revisão da Portaria MS/GM no 687, de 30 de março de 2006. Secretaria de Vigilância à Saúde, Secretaria de Atenção à Saúde. Ministério da Saúde, 2014.

BRETLAND, R. J.; THORSTEINSSON, E. B. Reducing workplace burnout: The relative benefits of cardiovascular and resistance exercise. PeerJ, v. 3, p. e891, 2015.

BURGARD, S. A.; LIN, K. Y. Bad jobs, bad health? How work and working conditions contribute to health disparities. American Behavioral Scientist, v. 57, n. 8, p. 1105-1127, 2013.

CICONELLI, R. M. et al. Brazilian-Portuguese version of the SF-36. A reliable and valid quality of life outcome measure. Rev Bras Reumatol, v. 39, n. 3, p. 143-50, 1999.

D'ANGELO, S. et al. Job dissatisfaction and the older worker: baseline findings from the Health and Employment After Fifty study. Occup Environ Med, v. 73, n. 8, p. 512-519, 2016.
D'ETTORRE, G.; GRECO, M. Healthcare work and organizational interventions to prevent work-related stress in Brindisi, Italy. Safety and health at work, v. 6, n. 1, p. 35-38, 2015.

DEL POZO-CRUZ, B. et al. An occupational, internetbased intervention to prevent chronicity in subacute lower back pain: a randomised controlled trial. Journal of rehabilitation medicine, v. 44, n. 7, p. 581, 2012.

ELLIOTT, K; SHELLEY, K. Impact of employee assistance programs on substance abusers and workplace safety. Journal of Employment Counseling, v. 42, n. 3, p. $125-132,2005$.

FAIRBANK, J. C. et al. The Oswestry low back pain disability questionnaire. Physiotherapy, v. 66, n. 8, p. 271-273, 1980 .

FISKER, A. et al. Early coordinated multidisciplinary intervention to prevent sickness absence and labour market exclusion in patients with low back pain: study protocol of a randomized controlled trial. BMC musculoskeletaldisorders, v. 14, n. 1, p. 93, 2013.

FREITAS, K. P. N. et al. Occupational low back pain and postural in the sitting position: labor kinesiotherapy. Rev. dor, São Paulo, v. 12, n. 4, p. 308-313, Dec. 2011.

FRESE, E. M.; FICK, A.; SADOWSKY, H. S. Blood pressure measurement guidelines for physical therapists. Cardiopulmonary physical therapy journal, v. 22, n. 2, p. 5, 2011.

GANESAN, S. et al. Prevalence and Risk Factors for Low Back Pain in 1,355 Young Adults: A Cross-Sectional Study. Asian Spine Journal, v. 11, n. 4, p. 610-617, 2017.

GOUVEIA, N. et al. Prevalence and social burden of active chronic low back pain in the adult Portuguese population: results from a national survey. Rheumatolog yinternational, v. 36, n. 2, p. 183197, 2016.

HENSCHKE, N.; KAMPER, S. J.; MAHER, C. G. The epidemiology and economic consequences of pain. In: Mayo Clinic Proceedings. Elsevier, 2015. p. 139-147. 
HENSCHKE, N. et al. Understanding cultural influences on back pain and back pain research. Best Practice \& Research Clinical Rheumatology, 2017.

LUCKHAUPT, S. E. et al. Prevalence of obesity among US workers and associations with occupational factors. American journal of preventive medicine, $v$. 46, n. 3, p. 237-248, 2014.

MALTA, D. et al. Política Nacional de Promoção da Saúde, descrição da implementação do eixo atividade física e práticas corporais, 2006 a 2014. Revista Brasileira de Atividade Física \& Saúde, v. 19, n. 3, p. 286, 2014.

MASSELLI, M. R. et al. Índice Funcional de Oswestry após cirurgia para descompressão de raízes nervosas. FisioterMov, v. 20, n. 1, p. 115-22, 2007.

JUNG J.et al.Association between working time quality and self-perceived health: analysis of the 3rd Korean working conditions survey (2011). Annals of Occupational and Environmental Medicine, v. 29, n. 1, p. 55, 2017.

JUNIO, J. F. V. et al. Tecnologia X Saúde: Estudo Sobre a Ocorrência de Lesões Musculoesqueléticas em Universitários Usuários de Notebooks. Saúde e Pesquisa, v. 8, n. 1, p. 63-70, 2015.

KIM, Y.; PARK, J.; PARK, M. Creating a culture of prevention in occupational safety and health practice. Safety and health at work, v. 7, n. 2, p. 89-96, 2016.

MUNRO, B. H. Statistical methods for health care research. Lippincott Williams \& Wilkins, v. 1, 2005.

PEREIRA M. S. et al. A Relação entre as condições de trabalho e saúde dos estudantes trabalhadores. Saúde e Pesquisa, v. 9, n. 3, p. 525-535, 2017.

RUTANEN, R. et al. Effect of physical exercise on work ability and daily strain in symptomatic menopausal women: a randomized controlled trial. Work, v. 47, n. 2, p. 281-286, 2014.

SEIDL, E. M. F.; ZANNON, C. M. L. C. Qualidade de vida e saúde: aspectos conceituais e metodológicos. Cad. Saúde Pública, Rio de Janeiro, v. 20, n. 2, p. 580-588, Apr. 2004.
SHOJAEI, S. et al. A Multidisciplinary Workplace Intervention for Chronic Low Back Pain among Nursing Assistants in Iran. Asian spine journal, v. 11, n. 3, p. 419-426, 2017.

TUNG, C. et al. An analysis of healthy workplace accreditation and health promotion efforts based on employees' perspectives. Archives of Environmental \& Occupational Health, p. 1-8, 2017.

VIGATTO, R. et al. Development of a Brazilian Portuguese version of the Oswestry Disability Index: cross-cultural adaptation, reliability, and validity. Spine, v. 32, n. 4, p. 481-486, 2007.

WAONGENNGARM, P.; RAJARATNAM, B. S.; JANWANTANAKUL, P. Perceived body discomfort and trunk muscle activity in three prolonged sitting postures. Journal of physical therapy science, v. $27, \mathrm{n}$. 7, p. 2183-2187, 2015.

WARE JR, J. E.; SHERBOURNE, C. D. The MOS 36item short-form health survey (SF-36): I. Conceptual framework and item selection. Medical care, p. 473-483, 1992.

YORKS, D. M.; FROTHINGHAM, C. A.; SCHUENKE, M. D. Effects of group fitness classes on stress and quality of life of medical students. The Journal of the American Osteopathic Association, v. 117, n. 11, p. e17, 2017

ZENK, R. et al. Spine loading in automotive seating. Applied ergonomics, v. 43, n. 2, p. 290-295, 2012.

Recebido em: 2017-08-22 Aceito em: 2017-12-05 